Proceeding Series of the Brazilian Society of Computational and Applied Mathematics

\title{
Análise Intervalar Aplicada ao Projeto de Controlador Robusto para Regulação da Velocidade de um Sistema de Geração em Escala Reduzida
}

Anderson de França Silva ${ }^{1}$

Programa de Pós-Graduação em Engenharia Elétrica, UFPA, Belém, PA

Cleyson Amorim Costa ${ }^{2}$

Setor de Desenvolvimento de Drive Seriados, WEG S.A., Jaraguá do Sul, SC

Carlos Tavares da Costa Junior ${ }^{3}$

Programa de Pós-Graduação em Engenharia Elétrica, UFPA, Belém, PA

Walter Barra Junior

Programa de Pós-Graduação em Engenharia Elétrica, UFPA, Belém, PA

Antonio da Silva Silveira ${ }^{5}$

Programa de Pós-Graduação em Engenharia Elétrica, UFPA, Belém, PA

Resumo. Neste artigo é apresentado o estudo dos conceitos da análise intervalar para projeto de um controlador robusto aplicado ao problema de regulação da velocidade de um gerador síncrono. Foi desenvolvido um controlador capaz de manter uma menor degradação do desempenho face às incertezas paramétricas do sistema. Os resultados confirmam o bom desempenho de controlador projetado.

Palavras-chave. Análise Intervalar, Regulador de Velocidade, Controle Robusto, Turbinas Hidráulicas, Geração de Energia.

\section{Introdução}

Em sistemas de hidrogeração de energia elétrica, um dos componentes fundamentais é o Regulador de Velocidade (RV). O controle de velocidade das turbinas hidráulicas

\footnotetext{
1 silva.anso@gmail.com

2 cleyson.am@gmail.com

3 cartav@ufpa.br

4 walbarra@ufpa.br

5 asilveira@ufpa.br
} 
efetuado pelo RV é um processo complexo que é influenciado, principalmente, pelas dinâmicas hidráulica e eletromecânica do gerador. Tratando-se da dinâmica hidráulica, um dos parâmetros que mais influencia a dinâmica do sistema é a altura da queda hidráulica. A altura da queda hidráulica é representada pela diferença entre os níveis do reservatório e da turbina. Sabe-se que o desempenho das turbinas hidráulicas é influenciado pelas características da coluna de água que alimenta a turbina [2]. Essa influência ocorre devido ao princípio físico que envolve a hidrogeração, que é o aproveitamento da energia potencial gravitacional da água contida em uma represa elevada ou proveniente de uma queda d'água natural. Portanto, torna-se necessário o projeto de RV's que mantenham o desempenho desejado diante de incertezas representadas por uma faixa de valores nos parâmetros do modelo da planta. Neste estudo, a variação do nível do reservatório será considerada como a incerteza paramétrica no sistema hidrogerador.

Em pesquisas sobre sistemas dinâmicos com incertezas paramétricas, as técnicas que lidam com esse problema vêm sendo estudadas extensivamente desde os anos 70. Nesse contexto, estratégias de controle que visam implementar algoritmos de controle adaptativo, preditivo e fuzzy vêm sendo largamente estudadas para resolução de problemas de controle de sistemas com incertezas paramétricas. Contudo, as estratégias do tipo adaptativas e fuzzy, ao mesmo tempo em que são capazes de oferecer desempenhos eficientes e satisfatórios, podem tornar o processo de implementação e operação mais complexo [1]. Em [4], plantas incertas são representadas por funções de transferências que possuem coeficientes pertencentes a intervalos reais e a alocação robusta de polos de malha fechada é desenvolvida buscando-se alocá-los robustamente numa região especificada. Portanto, as incertezas paramétricas de um modelo matemático são analisadas no projeto de controladores através de conceitos da análise intervalar.

Neste trabalho, é apresentado o projeto e a implementação experimental de uma estratégia de controle robusto via análise intervalar. A estratégia é aplicada ao projeto do regulador de velocidade robusto. Os testes para validação foram realizados, experimentalmente, em um sistema em escala reduzida com um gerador síncrono de 10 kVA.

\section{Propósito}

Dentro do âmbito da hidrogeração, uma discussão importante consiste na forma com a qual são feitos o uso e a conservação dos recursos hídricos. O uso da água para geração de energia deve causar um menor impacto ambiental, devendo-se considerar em todo projeto, tanto de grandes quanto pequenas centrais hidrelétricas, não comprometer a sustentabilidade do meio-ambiente onde localiza-se a bacia hídrica. Sendo assim, com a finalidade de reduzir o consumo de água necessário em turbinas hidráulicas, foi desenvolvido um controlador robusto para ser aplicado como RV.

Outro propósito para a realização deste trabalho é a possibilidade de sintetizar controladores robustos cuja implementação seja mais simples. Pois, com o auxílio da teoria intervalar aplicada ao projeto de controladores é possível obter um controlador robusto que apresente maior facilidade na implementação em sistemas digitais quando comparado com técnicas de controle robusto avançado como as adaptativas [6]. 


\section{Metodologia}

Nesta seção estão descritos os métodos utilizados para desenvolvimento deste trabalho. Inicialmente é apresentado o sistema de geração em estudo. Em seguida, é descrito o modelo matemático obtido com auxílio da análise intervalar. Por fim, mostrase as estratégias de controle convencional e não convencional proposta para o projeto de controladores aplicados como reguladores de velocidade.

\subsection{Descrição do sistema em estudo}

Para validação da estratégia de controle proposta neste trabalho foi utilizado um sistema de geração em escala reduzida de $10 \mathrm{kVA}$, o qual é constituído por um motor $\mathrm{CC}$ de $9 \mathrm{~kW}$, um gerador síncrono, um volante metálico de acoplamento entre gerador e motor, um circuito indutivo para simular uma linha de transmissão, um painel que contem toda instrumentação de automação, controle e acionamento do sistema. O grupo gerador composto pelo motor CC e a máquina síncrona é denominado Micromáquina [5]. Tal planta didática, ilustrada na Figura 1, foi dimensionada de forma que seus parâmetros elétricos e mecânicos em valores por unidade (p.u.) se assemelhassem aos valores de um sistema de grande porte.

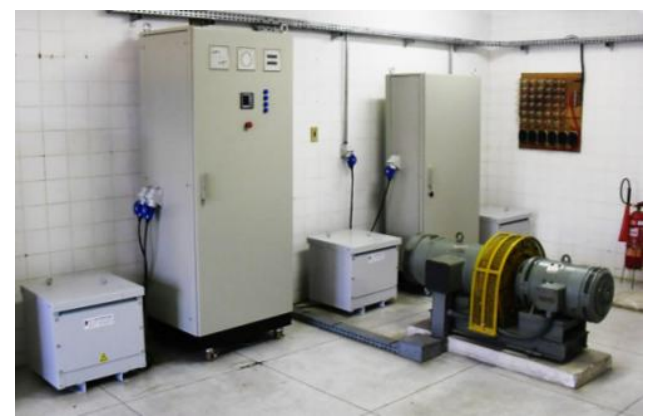

Figura 1: Sistema de geração em escala reduzida.

\subsection{Modelo Matemático}

Na modelagem matemática utilizada foram levantados modelos tanto com parâmetros fixos quanto com parâmetros intervalares do sistema em estudo. $\mathrm{O}$ modelo com parâmetros fixos foi obtido com objetivo de utilizá-lo para o projeto de um controlador convencional. Portanto é considerado um ponto de operação fixo. No segundo caso, foi inserida uma variação paramétrica no sistema modificando-se o ponto de operação, o qual é representado por um intervalo real. Esta variação paramétrica resulta em uma planta do tipo intervalar, a qual é utilizada para o projeto do controlador robusto intervalar.

As equações que expressam a dinâmica deste sistema em estudo estão relacionadas principalmente com o comportamento dinâmico do servoposicionador, da turbina hidráulica e das partes rotativas do grupo motor-gerador . A dedução do modelo matemático do sistema é fornecida de maneira mais detalhada em [2] e [5]. A função de transferência da planta é representada pelas Equações (1) e (2), com a devida substituição dos valores nominais da 
planta [6], os quais estão na Tabela 1. Este modelo é utilizado para o projeto do controlador considerando-se um ponto de operação fixo.

Tabela 1: Valores dos parâmetros físicos.

\begin{tabular}{|c|c|c|}
\hline Parâmetro Físico & Valor do Parâmetro & Nome do Parâmetro \\
\hline$T_{w}$ & $1,54 \mathrm{~s}$ & Tempo de partida da água \\
\hline$H$ & $4,29 \mathrm{~s}$ & Constante de Inércia \\
\hline$t_{g}$ & $2,8 \mathrm{~s}$ & $\begin{array}{c}\text { Constante de tempo entre a } \\
\text { válvula distribuidora e } \\
\text { servomotor }\end{array}$ \\
\hline$B$ & $0,03482 \mathrm{~N} \cdot \mathrm{m} / \mathrm{rad} / \mathrm{s}$ & Constante de Amortecimento \\
\hline
\end{tabular}

$$
\begin{gathered}
G(s)=\frac{\frac{-1}{H \times T_{g}}\left(s-\frac{1}{T_{w}}\right)}{\left(s+\frac{1}{T_{g}}\right)\left(s+\frac{2}{T_{w}}\right)\left(s+\frac{B}{2 \times H}\right)} \\
G(s)=\frac{-0,08325 s+0,05406}{s^{3}+1,66 \mathrm{~s}^{2}+0,4705 \mathrm{~s}+0,001882} \\
T_{w}=\frac{L P_{m}}{a_{g} k_{p} H_{0}^{5 / 2}}
\end{gathered}
$$

O modelo matemático sugerido para inserir a variação do ponto de operação do sistema em estudo, parte das equações que estão relacionadas com a dinâmica da turbina [6]. Com a Equação (3) é possível alterar a dinâmica da turbina a partir da modificação da variável $H_{0}$. A partir do comportamento apresentado pela variação no nível dos reservatórios das usinas hidrelétricas é possível considerar uma faixa de valores razoável para a variável $H_{0}$. Sendo assim, optou-se por considerar uma variação da queda hidráulica $H_{0}$, de $70 \%$ a $100 \%$ de sua capacidade. A partir da Equação (3) obtêm-se na Tabela 2 os valores para a constante de tempo da turbina em função de $H_{0}$.

Tabela 2: Valores da constante $T_{w}$ para cada ponto de operação.

\begin{tabular}{|c|c|}
\hline $\begin{array}{l}\text { Nível do } \\
\text { Reservatório }\end{array}$ & $\begin{array}{l}\text { Constante de Tempo } \\
\text { da Turbina }\end{array}$ \\
\hline$H_{0}=1 p u$ & $T_{w}=1,54 \mathrm{~s}$ \\
\hline$H_{0}=0,7 \mathrm{pu}$ & $T_{w}=3,756 \mathrm{~s}$ \\
\hline
\end{tabular}

Com os valores contidos na Tabela 1 é possível definir o intervalo real da Equação (4), o qual representa as incertezas paramétricas do sistema.

$$
T_{w}=[1,54,3,756]
$$

Para inserir tais incertezas paramétricas no sistema, são aplicados os valores da Equação (4) na forma genérica da planta representada pela Equação (1), que resulta em uma função de transferência da planta intervalar da Equação (5). 


$$
[G(s)]=\frac{-0,08325 s+[0.022162,0.054058]}{s^{3}+[0.893621,1.659902] s^{2}+[0.193760,0.470542] s+[0.000772,0.001882]}
$$

\subsection{Sintonia dos Controladores}

Esta subseção tem como objetivo apresentar dois métodos utilizados para se fazer a sintonia dos parâmetros dos controladores. (i) No método convencional, um controlador PI é projetado através da alocação polinomial de polos, a partir da solução de uma equação Diofantina. (ii) Já no método não convencional, é projetado um controlador intervalar robusto projetado via alocação robusta de polos a partir da solução de uma equação Diofantina intervalar [6]. O propósito destes controladores é fazer a regulação da velocidade de uma turbina hidráulica emulada por um motor $\mathrm{CC}$ de $9 \mathrm{~kW}$ com o intuito de controlar a potência desenvolvida pela turbina (motor CC).

\subsubsection{Controlador Convencional}

A metodologia utilizada para o projeto do controlador PI por alocação polinomial de polos a partir da solução da equação Diofantina não intervalar, fornece o controlador convencional. A planta utilizada neste projeto segue o modelo representado na Equação (2), o qual é especificado considerando-se um ponto de operação fixo. Assim, o controlador que resulta deste projeto é denominado RV Convencional. A solução da equação Diofantina resume o problema de alocação de polos.

Contudo, é necessário transformá-la num sistema de equações algébricas lineares. Assim, poderá ser reescrita como a equação linear a seguir,

$$
M x=P
$$

Sendo $M$ a matriz de Sylvester associada aos coeficientes dos polinômios da planta expressa pela Equação (2), $P$ o vetor associado aos coeficientes do polinômio desejado em malha fechada e $x$ o vetor associado aos coeficientes do controlador [4].

\subsubsection{Controlador Intervalar}

De forma análoga, para um sistema com incerteza intervalar, onde os coeficientes dos polinômios da planta e do polinômio desejado de malha fechada descrevem quantidades incertas com respeito à dinâmica do sistema a ser controlado, a Equação (5) pode ser claramente reescrita como uma equação linear intervalar [4].

$$
[M] x=[P]
$$

O conjunto solução de (6) apresenta todos os controladores para a qual existe uma planta $M \in[M]$ e um polinômio característico desejado $P \in[P]$ que atendam as especificações de desempenho desejado para o sistema em malha fechada. Entretanto, a grande variação nos parâmetros do controlador $x$ provoca fragilidade no mesmo, a ponto que pequenas variações nos parâmetros do controlador ou da dinâmica da planta podem deteriorar o desempenho do sistema em malha fechada. Uma das formas de diminuir a fragilidade do controlador intervalar, é selecionando o controlador central do conjunto de $[x]$ a partir da equação a seguir [4], 


$$
x_{c}=\frac{1}{2}\left(x^{-}+x^{+}\right)
$$

A Equação (7) fornece os seguintes valores para os parâmetros do controlador central,

$$
x_{\text {central }}=\left[\begin{array}{l}
0.754093286231937 \\
0.992333560782856 \\
0.291805142125286 \\
0.011968002650000 \\
1.000000000000000 \\
1.610045039018952 \\
0.938215104609388 \\
0.299306731883888
\end{array}\right]
$$

Cujos coeficientes admitem maior variação nos parâmetros do sistema, sem que o desempenho de malha fechada seja comprometido. Este controlador denomina-se: RV Central.

\subsubsection{Discretização dos Controladores}

Nas subseções anteriores mostrou-se as estratégias de controle utilizadas para o projeto do Regulador de Velocidade. Fica evidente que o resultado dos métodos utilizados irão fornecer controladores no domínio da frequência contínuo. No entanto, para o sistema de geração em estudo, é necessário ser projetado um RV digital. Em [6] são descritos detalhadamente a forma de discretização dos controladores com estrutura canônica RST [3], e também são fornecidos os parâmetros dos controladores digitais.

\section{Resultados Experimentais}

Foi realizado um teste de inserção de carga com o sistema de geração operando de forma isolada, isto é, alimentando unicamente as cargas conectadas nos terminais do gerador síncrono de $10 \mathrm{kVA}$. Na Figura 2 são ilustradas as respostas do sinal de velocidade e controle comparando o RV Convencional com o RV Central. Após a inserção de um degrau positivo de carga de 0,12 pu, nota-se que, para $100 \%$ da coluna de água, os dois controladores operam em condições satisfatórias de desempenho tanto para a resposta da velocidade quanto para o esforço de controle. Porém, quando submetidos a $70 \%$ do nível do reservatório, os controladores apresentam um tempo maior para atingir o erro de regime nulo na resposta da velocidade. Contudo, destaca-se que o sinal de controle do controlador Central, o qual é capaz de regular a velocidade com um menor esforço de controle, alcança um resultado mais satisfatório do que o Convencional devido ao fato de utilizar uma menor quantidade de água aplicada á turbina. Porquanto, a amplitude do esforço de controle representa a porcentagem de abertura da válvula distribuidora da turbina hidráulica. 

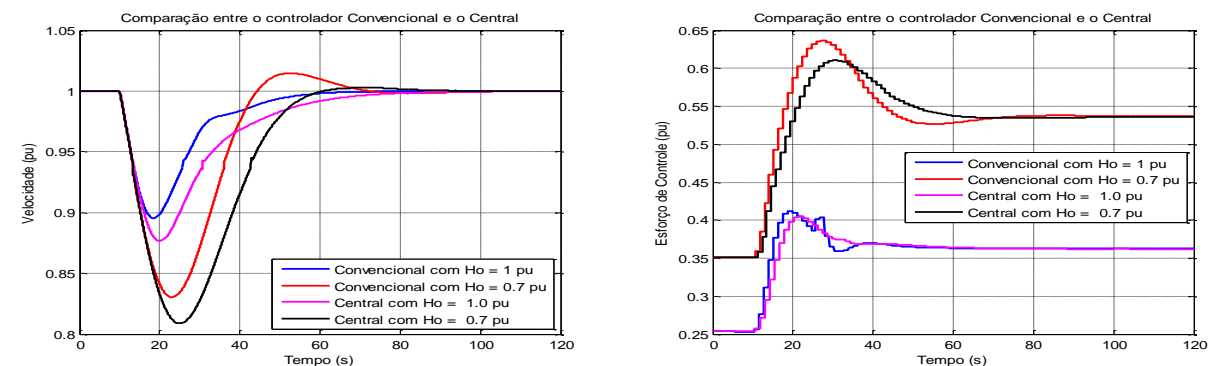

Figura 2: Teste de Inserção de Carga realizado aplicando-se um degrau positivo de carga.

\section{Conclusão e Considerações Finais}

Uma das grandes vantagens da aplicação do RV robusto ao problema de regular a velocidade de turbinas hidráulicas, é comprovada no esforço de controle utilizado por este controlador quando submetido à uma variação do ponto de operação. O RV robusto alcança a regulação da velocidade com uma menor quantidade de água sendo aplicada à turbina. Provocando a redução no consumo de água utilizado para gerar a energia elétrica, fazendo-se um melhor uso desse valioso recurso natural.

A partir dos resultados apresentados, conclui-se que a estratégia de controle robusto, com abordagem paramétrica via análise intervalar, apresenta-se como uma vantajosa forma de se projetar controladores aplicados como reguladores de velocidade de turbinas hidráulicas.

\section{Referências}

[1] W. Barra Junior; J. A. L Barreiros; C. T. Costa Júnior; A. M. D. Ferreira - Controle Fuzzy Aplicado à Melhoria da Estabilidade Dinâmica em Sistemas Elétricos de Potência - Revista Controle \& Automação/Vol.16 no.2/Abril, Maio e Junho (2005).

[2] P. Kundur- Power System Stability and Control - McGraw-Hill, 1994.

[3] I.D Landau, \& G. Zito - Digital Control Systems: Design, Identification and Implementation - Springer, (2006).

[4] A. D. S. Lordelo - Análise e Projeto de Controladores Robustos por Alocação de Pólos via Análise Intervalar - Tese de Doutorado FEEC - UNICAMP, (2004).

[5] P. S. Nascimento Filho - Investigação de estratégias de controle digital para regulação de velocidade e emulação da dinâmica de turbinas hidráulicas, com implementação e testes experimentais em uma micromáquina de 10kva - Dissertação de Mestrado. UFPA, (2011).

[6] A. F. Silva - Projeto e Avaliação Experimental de Controlador Robusto Intervalar para Regulação de Velocidade em um Sistema de Geração em Escala Reduzida - Trabalho de Conclusão de Curso. UFPA, (2014). 